\title{
Analysis of Omni-directivity Error of Electromagnetic Field Probe using Isotropic Antenna
}

\author{
Rene Hartansky \\ Institute of Electrical Engineering, Faculty of Electrical Engineering and Information Technology, Slovak Technical Univer- \\ sity, Ilkovicova 3, 81219 Bratislava, Slovakia, rene.hartansky@stuba.sk
}

\begin{abstract}
This manuscript analyzes the omni-directivity error of an electromagnetic field (EM) probe and its dependence on frequency. The global directional characteristic of a whole EM probe consists of three independent directional characteristics of EM sensors - one for each coordinate. The shape of particular directional characteristics is frequency dependent and so is the shape of the whole EM probe's global directional characteristic. This results in systematic error induced in the measurement of EM fields. This manuscript also contains quantitative formulation of such errors caused by the shape change of directional characteristics for different types of sensors depending on frequency and their mutual arrangement.
\end{abstract}

Keywords: Electromagnetics, field sensor, error of measurement, Cartesian coordinates, spherical coordinates, coordinates transformation.

\section{INTRODUCTION}

In electromagnetic compatibility measurements [1, 2], wireless sensor networks $[3,4,5]$ and other technical areas it is necessary to measure the intensity of electrical fields with adequate accuracy. The measurement has to be performed over a sufficient frequency and voltage range, whilst the direction of the measured (incident) wave is not completely known. In the measuring space a large number of reflections and other impairments can occur which affect the result in the measured point. Based on these facts, for measuring an EM field it is necessary to use such field probes whose output quantity is independent of the direction of the incident wave. The probe output also has to be frequency independent (at least to a sufficient extent). Since the measuring part of a probe (consisting of several sensors) takes a certain volume of the measured space, the sensor output will depend on the averaged value of the sensed electrical field in the space volume taken by the probe [6]. In addition, real EM probes have non-ideal characteristics. The produced EM probes have their parameters specified for the working range of frequencies, but their omnidirectivity is usually specified only for one frequency: e.g., $100 \mathrm{MHz}$. The practical use of a particular EM probe for a given EMC measurement is determined by its amplitude, frequency and omni-directivity characteristics. From the available commercial information it is not possible to determine the omni-directivity of a given EM probe and its effect on the output quantity. The presented analysis deals with the analytical expression of frequency dependence of EM probe omni-directivity for two most commonly used types of sen-

DOI: $10.1515 / \mathrm{msr}-2016-0036$ sors (creating the probe): resistive dipoles and finite length dipoles.

\section{DIRECTIONAL CHARACTERISTIC OF PROBE SENSORS}

Most EM field probes consist of three spatially arranged field sensors. Sensors are located in space so they create an orthogonal system, that means they are placed on the axes of a coordinate system or on the sides of a trihedral prism [7]. Each sensor has its own directional characteristic, which can be described analytically, e.g. [8, 9] using directivity. Sensor directivity is a function representing the ratio of the radiated EM field in the given direction to the average value over 4 $\pi$ [ste]. Directivity is expressed using two orthogonal planes: the E plane and the $\mathrm{H}$ plane using a spherical coordinate system. In order to determine the overall probe directivity it is necessary to calculate the directivity of the individual sensors [7]. Directivity of individual sensors create directive characteristics in space which are mutually rotated while we do not know their analytical expressions in rotated form. In such cases the most effective way of expressing rotated directional characteristics of sensors is to use spherical coordinates. It means that directive angles $\theta$ and $\phi$ will also depend on a rotation of a directive characteristic in space. The directive characteristics of an EM probe can be imagined as a 3D body whose surface can be described as

$$
f(r, \vartheta, \varphi)=0 .
$$

In the following it is necessary to note that each surface point of a 3D body fulfills (1). The axes of a coordinate system in 




Fig.1. Rotation of axes in coordinate systems.

which a 3D body is placed are denoted as: $x, y, z$. Then the relationship between the Cartesian and spherical coordinate system can be expressed as

$$
\begin{aligned}
& x=r \sin \vartheta \cos \varphi \\
& y=r \sin \vartheta \sin \varphi \\
& z=r \cos \vartheta .
\end{aligned}
$$

The inverse relationships are

$$
\begin{aligned}
r & =\sqrt{x^{2}+y^{2}+z^{2}} \\
\vartheta & =\arccos \left(\frac{z}{\sqrt{x^{2}+y^{2}+z^{2}}}\right) \\
\varphi & =\arctan \frac{y}{x} .
\end{aligned}
$$

Let us assume that a 3D body is rotated arbitrarily in space. Such arbitrary rotation can be defined as follows:

1. rotation about $z$ axis is determined by angle $\alpha$;

2. rotation about $\bar{\xi}$ axis lying in plane $x y$ is determined by angle $\beta$. This axis includes axis $x$, angle $\gamma$.

Based on Fig.1, the rotated axis from step 2 will include auxiliary axis $\xi$ angle $\delta$, and holds: $\gamma=\alpha+\delta$. Let us describe Fig. 1 in detail. The basis coordinate system has axes denoted as: $x, y, z$. In this coordinate system we place a $3 \mathrm{D}$ body. Let us rotate the 3D body around axis $z$ by angle $\alpha$. Further let us assume that the $3 \mathrm{D}$ body has its own rotating system which moves as the body moves. This moving coordinate system rotates with regard to the original coordinate system to the position: $\xi, \eta, \zeta$, (axis $\zeta$ is identical to axis $z$ ). Next let us perform the second rotation of a 3D body by angle $\beta$ around axis $\bar{\xi}$. The rotating system is fixed to the 3D body and gets to the position which will be denoted as a resulting coordinate system: $X, Y, Z$. This resulting coordinate system is not shown on the figure due to readability.

The basic idea of the solution is now very simple. If the 3D body (directional characteristic) has, in the Cartesian coordinate system $x, y, z$ analytical expression $g(x, y, z)=0$, then in the resulting coordinate system: $X, Y, Z$ it will have the identical analytical expression: $g(X, Y, Z)=0$. Only the coordinates have changed from $(x, y, z)$ to $(X, Y, Z)$. The formal expression of the function $g$ stays the same.

In order to find an analytical expression of coordinates $(X, Y, Z)$ let us choose point $P$, located on the surface of a 3D body, whose coordinates are: $P(x, y, z)$. If the 3D body is rotated around $z$ axis by angle $\alpha$, then point $P$ relocates to the new position $P^{\prime}\left(x^{\prime}, y^{\prime}, z^{\prime}\right)$. After the next rotation around $\bar{\xi}$ axis by angle $\beta$, the chosen point $P^{\prime}\left(x^{\prime}, y^{\prime}, z^{\prime}\right)$ relocates again to the position $P^{\prime \prime}\left(x^{\prime \prime}, y^{\prime \prime}, z^{\prime \prime}\right)$.

To avoid ambiguity we must stress the meaning of the notation of the coordinates used. Any coordinates without primes denote the physical position of the original point $P$. Any coordinates with a prime denote the physical position of point $P$ after the first rotation in the plane $x y$ by angle $\alpha$. Any coordinates with a double prime denote the physical position of point $P$ after the second rotation in the plane $\bar{\xi}_{z}$ by angle $\beta$.

Coordinates $(x, y, z),\left(x^{\prime}, y^{\prime}, z^{\prime}\right)$ and $\left(x^{\prime \prime}, y^{\prime \prime}, z^{\prime \prime}\right)$ are related to the original (reference) coordinate system. An arbitrary point $P$ can be also described in other Cartesian systems e.g., point $P^{\prime}$ has, in coordinate system $\xi, \eta, \zeta$, coordinates $P^{\prime}\left(\xi^{\prime}, \eta^{\prime}, \zeta^{\prime}\right)$. Similarly, point $P^{\prime \prime}$ has, in the same coordinate system coordinates $P^{\prime \prime}\left(\xi^{\prime \prime}, \eta^{\prime \prime}, \zeta^{\prime \prime}\right)$ and so on. The same principle can be used for setting a position in the coordinate system $X, Y, Z$. The original point $P$ has coordinates $P(X, Y, Z)$ and point $P^{\prime \prime}$ has coordinates $P^{\prime \prime}\left(X^{\prime \prime}, Y^{\prime \prime}, Z^{\prime \prime}\right)$. Therefore, it is possible to write the following equations

$$
\left(\begin{array}{l}
x \\
y \\
z
\end{array}\right)=\left(\begin{array}{l}
\xi^{\prime} \\
\eta^{\prime} \\
\zeta^{\prime}
\end{array}\right)=\left(\begin{array}{l}
X^{\prime \prime} \\
Y^{\prime \prime} \\
Z^{\prime \prime}
\end{array}\right) .
$$

The equation (4) expresses the equalities between Cartesian coordinates in selected Cartesian systems. These equations represent the fact that if we rotate point $P$ at the same time as we simultaneously rotate the coordinate system, the relative position of the chosen point with regard to the coordinate system stays the same.

If a chosen point (or the body) is rotated changing its coordinates relative to the fixed coordinate system and such change is relevant, such a process will be denoted as active rotation. If the coordinate system is rotated while the physical position of the chosen point is fixed, this process will be denoted as passive rotation.

Let us perform an active rotation of point $P$ by angle $\alpha$ around the $z$ axis. It is the first step of which body rotation consists. This rotation relocates point $P(x, y, z)$ to different physical location $P^{\prime}\left(x^{\prime}, y^{\prime}, z^{\prime}\right)$, which can be expressed as

$$
\left(\begin{array}{l}
x^{\prime} \\
y^{\prime} \\
z^{\prime}
\end{array}\right)=A(\alpha)\left(\begin{array}{l}
x \\
y \\
z
\end{array}\right)
$$

where $A(\alpha)$ is a matrix

$$
A(\alpha)=\left(\begin{array}{ccc}
\cos \alpha & -\sin \alpha & 0 \\
\sin \alpha & \cos \alpha & 0 \\
0 & 0 & 1
\end{array}\right)
$$


Let us continue in an active rotation of point $P$ around axis $\bar{\xi}$ by angle $\beta$. In this case the translation can be described simply only if we relate the Cartesian coordinates to the coordinate system $\bar{\xi}, \bar{\eta}, \bar{\zeta}$ :

$$
\left(\begin{array}{c}
\bar{\xi}^{\prime \prime} \\
\bar{\eta}^{\prime \prime} \\
\bar{\zeta}^{\prime \prime}
\end{array}\right)=B(\beta)\left(\begin{array}{c}
\bar{\xi}^{\prime} \\
\bar{\eta}^{\prime} \\
\bar{\zeta}^{\prime}
\end{array}\right),
$$

where $B(\beta)$ is a matrix

$$
B(\beta)=\left(\begin{array}{ccc}
1 & 0 & 0 \\
0 & \cos \beta & -\sin \beta \\
0 & \sin \beta & \cos \beta
\end{array}\right) .
$$

Let us perform the same steps described above for the passive rotation. In this case we will assume a physically static point in the moving coordinate system. The coordinate change of the chosen point takes place when the moving coordinate system rotates with respect to the original one. For a passive rotation of point $P$ around axis $z$ by angle $\alpha$ the following holds

$$
\left(\begin{array}{l}
\xi \\
\eta \\
\zeta
\end{array}\right)=A(-\alpha)\left(\begin{array}{l}
x \\
y \\
z
\end{array}\right)
$$

Relationship (9) describes the change of coordinate of the arbitrary physical point in space when the coordinate system rotates around axis $z$ by angle $\alpha^{1}$. Formally (9) can be rewritten for one prime and two prime coordinates, so for the final position of point $P$ the following holds

$$
\left(\begin{array}{l}
\xi^{\prime \prime} \\
\eta^{\prime \prime} \\
\zeta^{\prime \prime}
\end{array}\right)=A(-\alpha)\left(\begin{array}{c}
x^{\prime \prime} \\
y^{\prime \prime} \\
z^{\prime \prime}
\end{array}\right)
$$

Let us now perform the second step of passive rotation - the rotation of a chosen point together with the coordinate system around axis $\bar{\xi}$ lying in the plane $x y$ by angle $\beta$ :

$$
\left(\begin{array}{c}
X \\
Y \\
Z
\end{array}\right)=B(-\beta)\left(\begin{array}{c}
\bar{\xi} \\
\bar{\eta} \\
\bar{\zeta}
\end{array}\right) .
$$

Equation (11) holds for an arbitrary point in space. The analogical relationship between coordinate systems denoted with Greek letters is

$$
\left(\begin{array}{c}
\bar{\xi} \\
\bar{\eta} \\
\bar{\zeta}
\end{array}\right)=C(-\delta)\left(\begin{array}{l}
\xi \\
\eta \\
\zeta
\end{array}\right)
$$

where $C(\delta)$ is a matrix

$$
C(\delta)=\left(\begin{array}{ccc}
\cos \delta & \sin \delta & 0 \\
-\sin \delta & \cos \delta & 0 \\
0 & 0 & 1
\end{array}\right)
$$

${ }^{1}$ Using the standard mathematical convention the rotation will be assume as positive when it is performed counterclock wise.
Now we have the mathematical apparatus ready to describe an active rotation between the original and the new coordinates of a chosen point. Let us take (10) and write its right hand side as a vector. Do the same with (12). By equating those two right hand sides we get the final (two prime) coordinates description

$$
\left(\begin{array}{l}
x^{\prime \prime} \\
y^{\prime \prime} \\
z^{\prime \prime}
\end{array}\right)=A(\alpha)\left(\begin{array}{l}
\xi^{\prime \prime} \\
\eta^{\prime \prime} \\
\zeta^{\prime \prime}
\end{array}\right)=A(\alpha) C(\delta)\left(\begin{array}{c}
\bar{\xi}^{\prime \prime} \\
\bar{\eta}^{\prime \prime} \\
\bar{\zeta}^{\prime \prime}
\end{array}\right) .
$$

It is convenient to express the right hand side of (14) by using (11) so we get ${ }^{2}$

$$
\left(\begin{array}{c}
\bar{\xi}^{\prime \prime} \\
\bar{\eta}^{\prime \prime} \\
\bar{\zeta}^{\prime \prime}
\end{array}\right)=B(\beta)\left(\begin{array}{c}
X^{\prime \prime} \\
Y^{\prime \prime} \\
Z^{\prime \prime}
\end{array}\right)
$$

Next we substitute (15) to (14) and by using (4) we get

$$
\left(\begin{array}{l}
x^{\prime \prime} \\
y^{\prime \prime} \\
z^{\prime \prime}
\end{array}\right)=A(\alpha) C(\delta) B(\beta)\left(\begin{array}{l}
x \\
y \\
z
\end{array}\right) \text {. }
$$

After multiplication and rearranging (16) we get

$A(\alpha) C(\delta) B(\beta)=$

$\left(\begin{array}{ccc}\cos (\alpha+\delta) & -\cos \beta \sin (\alpha+\delta) & \sin \beta \sin (\alpha+\delta) \\ \sin (\alpha+\delta) & \cos \beta \cos (\alpha+\delta) & -\sin \beta \cos (\alpha+\delta) \\ 0 & \sin \beta & \cos \beta\end{array}\right)$

$=S(\alpha, \beta, \gamma)$,

where

$$
\gamma=\alpha+\beta
$$

Then (16) simplifies to

$$
\left(\begin{array}{l}
x^{\prime \prime} \\
y^{\prime \prime} \\
z^{\prime \prime}
\end{array}\right)=S(\alpha, \beta, \gamma)\left(\begin{array}{l}
x \\
y \\
z
\end{array}\right)
$$

\subsection{Transformation of spherical coordinates}

The directivity characteristic is in the reference coordinate system described by (1)

$$
f(r, \vartheta, \varphi)=0 .
$$

A rotated 3D body firmly joined with the rotated coordinate system can be described using the same equation

$$
f(R, \Theta, \Phi)=0
$$

Now it is necessary to express new spherical coordinates: $R, \Theta, \Phi$ in equation (20) by means of the original coordinates $r, \vartheta, \varphi$. The following relationships have to be found

$$
R=R(r, \vartheta, \varphi) \quad \Theta=\Theta(r, \vartheta, \varphi) \quad \Phi=\Phi(r, \vartheta, \varphi)
$$

\footnotetext{
${ }^{2}$ For derivation of this equation we used an inverse notation of (11).
} 
The expression of coordinate $R$ is trivial

$$
R=r
$$

For the remaining two coordinates we can use transformations (3)

$$
\begin{aligned}
\Theta & =\arccos \left(\frac{Z}{\sqrt{X^{2}+Y^{2}+Z^{2}}}\right) \\
\Phi & =\arctan \left(\frac{Y}{X}\right) .
\end{aligned}
$$

Instead of equation $f(R, \Theta, \Phi)=0$ we use equation $h(X, Y, Z)=0$ whilst the following holds

$$
h(X, Y, Z)=f(R(X, Y, Z), \Theta(X, Y, Z), \Phi(X, Y, Z)) .
$$

Based on (19) the passive transformation between the new and the original coordinate system can be described as

$$
\left(\begin{array}{l}
x \\
y \\
z
\end{array}\right)=S(\alpha, \beta, \gamma)\left(\begin{array}{c}
X \\
Y \\
Z
\end{array}\right) .
$$

Let us replace in (25) Cartesian coordinates $(x, y, z)$ for spherical ones. Taking into account that $R=r$ we get

$$
\left(\begin{array}{c}
\sin \vartheta \cos \varphi \\
\sin \vartheta \sin \varphi \\
\cos \vartheta
\end{array}\right)=S(\alpha, \beta, \gamma)\left(\begin{array}{c}
X \\
Y \\
Z
\end{array}\right)
$$

By substituting (17) into (26) and after solving the formed equation we obtain the representation of Cartesian coordinates in the new coordinate system

$$
\begin{aligned}
X & =\cos (\gamma-\varphi) \sin \vartheta \\
Y & =\cos \vartheta \sin \beta-\cos \beta \sin \vartheta \sin (\gamma-\varphi) \\
Z & =\cos \vartheta \cos \beta+\sin \beta \sin \vartheta \sin (\gamma-\varphi)
\end{aligned}
$$

Next it is necessary in (27) to replace Cartesian coordinates for spherical ones by using (3)

$$
\begin{aligned}
& R=r \\
& \Theta(r, \vartheta, \varphi, \beta, \gamma)= \\
& \quad \arccos (\cos \beta \cos \vartheta+\sin \beta \sin \vartheta \sin (\gamma-\varphi)) \\
& \Phi(r, \vartheta, \varphi, \beta, \gamma)= \\
& \quad \arctan (\cot \vartheta \sec (\gamma-\varphi) \sin \beta-\cos \beta \tan (\gamma-\varphi))
\end{aligned}
$$

(28) enables us to rotate a 3D body (directional characteristic of sensors) in the course of angles $\beta$ and $\gamma$ when this body is mathematically described using a spherical coordinate system. (28) also enables us to very effectively rotate directional characteristics of several EM sensors in order to calculate the overall directional characteristic of an EM probe as well as its frequency response in its working region.

\section{OMNI-DIRECTIVITY ERRORS OF AN EM PROBE}

In [1] the authors searched for the mutual position of three EM sensors so their overall directional characteristic is spherical. Several sensor configurations were found which satisfied the assignment. Particular directional characteristics were approximated by toruses. They did not take into account the frequency dependent change of its directional characteristic.

In the following study we do not use the approximation of directional characteristics of EM probes (as was done in [1]), but we will use the mathematical derivation of directional characteristic as was done in, e.g.[9, 10]. Let us assume that an EM probe consists of three finite length dipoles - as sensors. The analytical expression of directivity of a single dipole can be found in [9]. In the case of far field radiation the following holds

$$
F(\Theta, \Phi, k h)=\frac{\cos (k h \cos \Theta)-\cos k h}{\sin \Theta},
$$

as

$$
D(\Theta, \Phi, k h)=4 \pi \frac{F(\Theta, \Phi, k h)}{\int_{0}^{2 \pi} \int_{0}^{\pi} \sin \Theta d \Theta d \Phi} .
$$

It can be observed that directivity equals to far field radiation divided by a constant. Therefore, next we will assume only the far field condition. (29) is expressed directly in spherical coordinates so transformation (28) can be applied. The $k h$ element in this case represents the ratio of the dipole physical length to wave length of the incident wave. Careful analysis of (29) reveals that by changing $k h$, the far field radiation pattern (and thus directional characteristic) is also changed. Therefore, it is necessary to divide it by its max value

$$
\begin{aligned}
& F_{N}(\Theta, \Phi, k h)= \\
& \frac{\cos (k h \cos \Theta)-\cos k h}{\sin \Theta} \frac{\sin \frac{\pi}{2}}{\cos \left(k h \cos \frac{\pi}{2}\right)-\cos k h} .
\end{aligned}
$$

Equation (31) represents the normalized radiation of a finite length dipole. For calculating the overall directional characteristic of an EM probe we have to use (31) three times and variables $\Theta$ and $\Phi$ are replaced by (28).

In order to obtain the omni-directive characteristic of an EM field probe we need 3 sensors - dipoles placed in space. This concept is analyzed in [1] and is not the topic of this manuscript. At this point we take only the results of [1]. One of the possible dipole configurations is their placement on the walls of a tetrahedral prism (see Fig.2 and Fig.3) so that the dipoles axes are suitably mutually rotated. Under such conditions the normalized radiation pattern of an EM probe is

$$
\begin{aligned}
& F_{N_{S}}(\vartheta, \varphi, k h)=\left\{F_{N}\left(\vartheta, \varphi, \beta=\frac{\pi}{2}-\frac{\arccos \frac{1}{3}}{2}, \gamma=0, k h\right)^{2}\right. \\
& +F_{N}\left(\vartheta, \varphi, \beta=\frac{\pi}{2}-\frac{\arccos \frac{1}{3}}{2}, \gamma=\frac{2}{3} \pi, k h\right)^{2}
\end{aligned}
$$




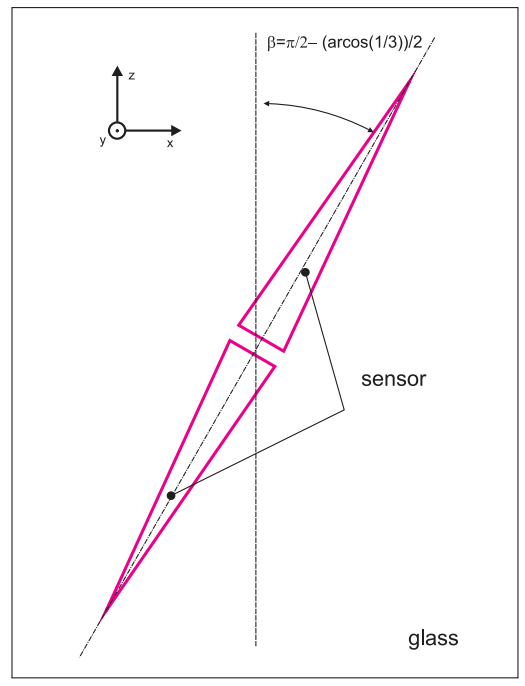

Fig.2. Sensor on glass substract.

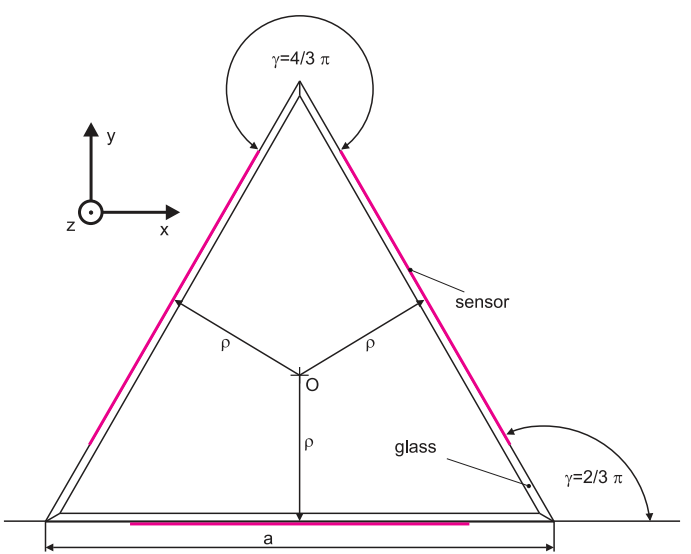

Fig.3. Probe contains three sensors.

$$
\left.+F_{N}\left(\vartheta, \varphi, \beta=\frac{\pi}{2}-\frac{\arccos \frac{1}{3}}{2}, \gamma=\frac{4}{3} \pi, k h\right)^{2}\right\}^{-\frac{1}{2}}
$$

The angles $\beta$ and $\gamma$ result from the spatial arrangement of dipoles and are taken from [1], taking into account the derivation of the transform formula given in subsection 2.1. In the ideal case (directional characteristic is independent of angles $\vartheta, \varphi$ and frequency) it holds that

$$
F_{N_{S}}(\vartheta, \varphi, k h)=\text { konst }
$$

by $[11,1]$

$$
F_{N_{S}}(\vartheta, \varphi, k h)=\sqrt{2}
$$

In reality this is not true and a frequency dependent change of the shape of omni-directional characteristic occurs. The relative error can be expressed as

$$
\delta_{S}(\vartheta, \varphi, k h)=\frac{F_{N_{S}}(\vartheta, \varphi, k h)-\sqrt{2}}{\sqrt{2}} 100 \% .
$$

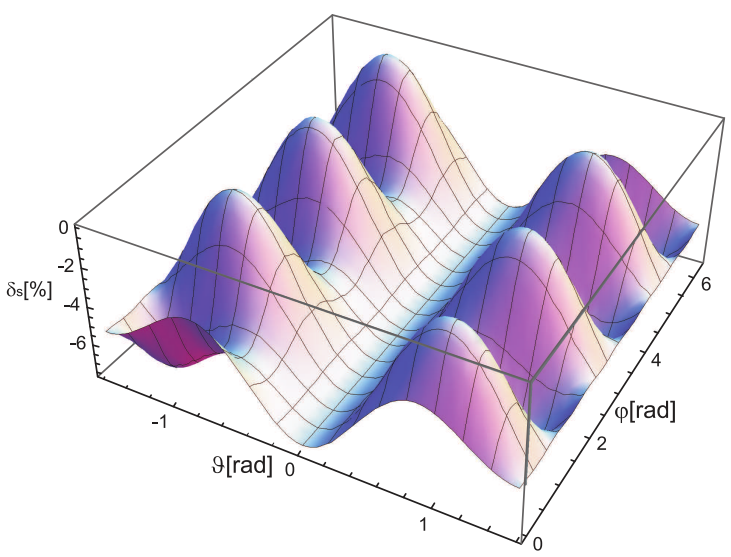

Fig.4. Relative error of omni-directivity for $k h=\pi / 2$.

A graphical representation of (35) - the relative error of omni-directivity is shown in Fig.4 and in Fig.5 for values of parameter $k h=\pi / 2$ and $k h=\pi / 3$, respectively.

For the sake of simplicity the relative error of omnidirectivity is plotted in Cartesian coordinates for spherical variables $\vartheta$ and $\varphi$. From Fig.4 it can be observed that for a value of $k h=\pi / 2$ the relative error of omni-directivity changes from $0 \%$ to $-7 \%$. With decreasing frequency the relative error of omni-directivity (compare Fig.4 and Fig.5) also decreases. We are interested in the relative error of omni-directivity depending on frequency. Calculating equation (35) enables us to quantify the magnitude of the relative error of omni-directivity in the whole frequency range of an EM probe ${ }^{3}$.

In order to analyze the relative error of omni-directivity in the whole frequency range it is necessary to calculate (35) depending on frequency. By plotting only extremes, Fig.6 shows the relative error of omni-directivity for an omnidirective probe. This error $\delta_{S}$ depends on angles $\vartheta, \varphi$ and the frequency is determined by parameter $k h$. This function reaches its peaks and valleys - as it is shown in Fig.4, Fig.5.

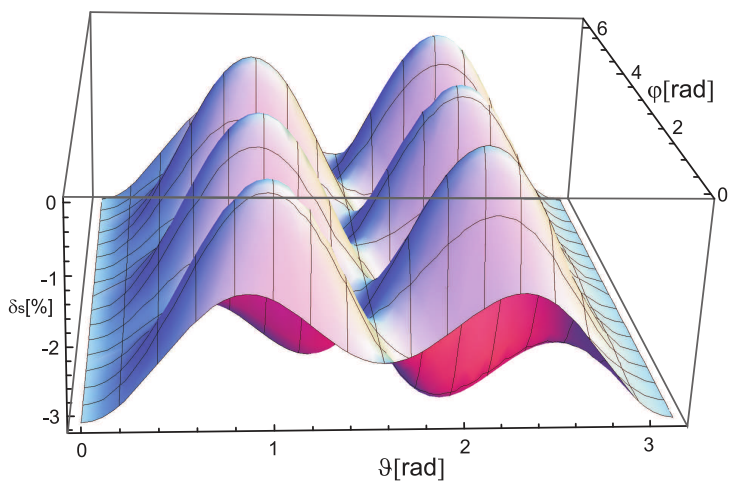

Fig.5. Relative error of omni-directivity for $k h=\pi / 3$.

\footnotetext{
${ }^{3}$ With regard to frequency characteristic of a sensor - coefficient of the effective length, we do not assume that sensor will be used for frequencies $k h>\pi / 2$.
} 


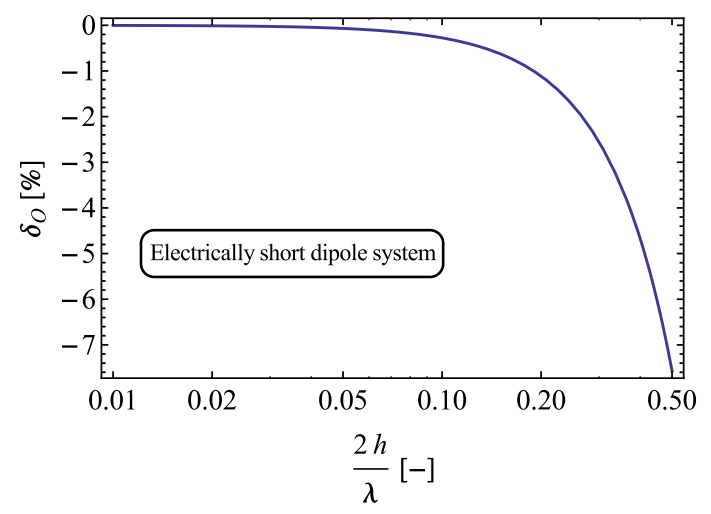

Fig. 6. Maximal negative relative error of omni-directivity relative to wave length for an omni-directive sensor created of finite length dipoles.

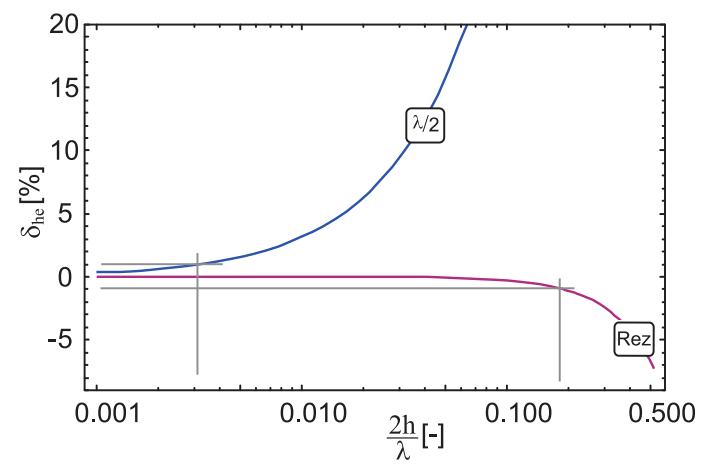

Fig.7. The relative error of the dipoles effective length.

The peaks of the function $\delta_{S}$ are always equal to 0 . Valleys (the negative extremes) reach values of percents depending on the ratio of dipole length and wave length of the incident wave.

From Fig.6 it can be observed that relative error of omnidirectivity is less than $-1 \%$, till the wave length of the incident wave $\lambda>10 \cdot h$. If the frequency of the incident wave rises ( $\lambda$ decreases), the relative error will fall sharply.

In practice we encounter the term amplitude-frequency characteristic (AFCH) of an EM field probe. This parameter plays a key role when choosing the probe. The higher the probe quality is the less ripple its $\mathrm{AFCH}$ should have in its working range. For example let us assume an AFCH of a finite length dipole and resistively loaded dipole $[12,13]$. From Fig.7 it is possible to observe that the AFCH of a finite length dipole reaches relative error $1 \%$ for the wave length of the incident wave $\lambda=66 \cdot h$. For higher frequencies this error rises. Now let us compare the measuring error caused by an AFCH ripple with measuring error caused by directional properties of a sensor (error of omni-directivity). By comparing Fig. 6 with Fig. 7 for max $\pm 1 \%$ of relative measuring error for omni-directional probe, it can be stated that a probe created from finite length dipoles will perform acceptably only for the wave lengths of the incident wave greater than $66 \cdot h$ (physical dimension of a sensor divided by 0.03 ).
The outcome is that the error of omni-directivity is within the working region of a probe marginal.

Let us continue with analysing the error of omni-directivity for a probe created from resistively loaded dipoles. The analytical expression of directional characteristic of one dipole can be found e.g., in [10]. The far field radiation function is

$F(\Theta, \Phi, k h)=$

$\frac{1}{k h \sin ^{3} \Theta}\left\{-j k h \sin ^{2} \Theta+\left(1+\cos ^{2} \Theta\right)\right.$

$-[2 j \cos \Theta \sin (k h \cos \Theta)$

$\left.\left.+\left(1+\cos ^{2} \Theta\right) \cos (k h \cos \Theta)\right]\right\} e^{-j k h}$.

Similarly, for a probe composed of finite length dipoles the directional characteristic of a resistively loaded dipole is frequency dependent, namely on the parameter $k h$. From (35) it is clear that with a frequency change the shape of the field radiation function also changes.

Let us focus now on the changes of the directional characteristic. Similarly to the previous case let us rearrange (31) the following way

$$
F_{N}^{R D}(\Theta, k h)=\frac{F(\Theta, k h)}{F\left(\frac{\pi}{2}, k h\right)} .
$$

Equation (37) represents a normalized far field radiation function of a resistively loaded dipole. Let us assume that an EM field probe consists of three resistively loaded dipoles, located in space as described in [1]. In order to rotate the directional characteristics of the individual dipoles in space it is necessary to transform space angles by applying (28). To calculate a normalized radiation intensity pattern of the EM probe we use (32), where instead of function $F_{N}$ we use $\dot{F}_{N}^{R D}$.

In the ideal case the radiation diagram of an EM probe should not be dependent on angles $\vartheta, \varphi$ and frequency. Such a function has the form

$$
F_{N_{S}}^{R D}(\vartheta, \varphi, k h)=\sqrt{2} .
$$

In practice the directional characteristic of a probe created from 3 resistively loaded dipoles has ripples and omnidirectivity errors occur. Based on the previous analysis the dependence of relative omni-directivity error on frequency in the whole frequency range can be plotted. In Fig.8 the relative error of omni-directivity for an EM probe is shown. Similarly to the case of finite length dipoles, in this case the radiation pattern is also frequency dependent, which results in an omnidirectivity error. By comparing Fig.8 and Fig.6 we can conclude that the shape of the two curves is practically identical; the only change is in the magnitude.

In spite of the fact that the resistively loaded dipole has much greater bandwidth, the fall of its AFCH by $1 \%$ happens for wave length $\lambda=10 \cdot h$ (see Fig.7). 




Fig. 8. Relative error of omni-directivity of an EM probe created from resistively loaded dipole vs. dipole wave length.

The directional characteristic of an EM probe created from resistive dipoles changes for wave length of the incident wave $\lambda=10 \cdot h$ (for $1 \%$ of measuring error). Based on these two facts, it is possible to state that EM probe created from resistively loaded dipoles will have an acceptable frequency response and omni-directivity for wave lengths greater than $10 \cdot h$ (physical dimension of a sensor divided by 0.2 ). The omni-directivity error in the working range has to be taken into account.

\section{CONCLUSION}

Based on the previous analysis we can conclude that an EM probe composed of resistively loaded dipoles has approximately 6 times greater bandwidth than a probe composed of finite length dipoles (assuming the same physical length of sensors). The practical utilization of a probe is limited by the AFCH together with the omni-directivity error. The omnidirectivity error can be neglected for finite length dipoles, since it starts to be significant for higher frequencies than the upper usable frequency of this EM probe. When designing an EM probe composed of resistively loaded dipoles and determining its usable bandwidth, both the omni-directivity error and the fall of $\mathrm{AFCH}$ for high frequencies have to be taken into account.

\section{ACKNOWLEDGEMENT}

This work was supported by the Slovak Research and Development Agency under the contract No. APVV-14-0076 and by the project VEGA 1/0431/15. Special thanks to Martin Konopka for transforming the coordinate systems.

\section{REFERENCES}

[1] Bittera, M., Kovac, K., Smiesko, V. (2002). Development of hf e-field probe with the frequency independent isotropy. In Radioelektronika 2002: 12th International Scientific Conference, May 14-16, 2002, 282-285.
[2] Zheng, G., Tang, J. (2016). Two-dimensional DOA estimation for monostatic MIMO radar with electromagnetic vector received sensors. International Journal of Antennas and Propagation, 2016, art. ID 2952539.

[3] Farkas, P., Mihalovic, M., Majchrak, S., Rakus, M. (2009). One experiment with wireless senzor network. In Telecommunications and Signal Processing: 32nd International Conference (TSP 2009), August 26-27, 2009. Budapest: Asszisztencia Szervezö Kft.

[4] Kenyeres, J., Sajban, S., Farkas, P., Mihalovic, M., Majchrak, S., Rakus, M. (2010). Indoor experiment with wsn application. In MIPRO 2010 : 33rd International Convention on Information and Communication Technology, Electronics and Microelectronics, May 24-28, 2010. IEEE, 836-866.

[5] Lomine, J., Morlaas, C., Imbert, C., Aubert, H. (2015). Dual-band vector sensor for direction of arrival estimation of incoming electromagnetic waves. IEEE Transactions on Antenna and Propagation 63 (8), 3662-3671.

[6] Slizik, J., Hartansky, R. (2013). Metrology of electromagnetic intensity measurement in near field. Kvalita Inovácia Prosperita (Quality Innovation Prosperity), 17 (1), 57-66.

[7] Bittera, M., Hartansky, R., Kovac, K. (2001). One way to provision of hf e-field sensor isotropy. Measurement Science Review, 1 (1), 73-76.

[8] Balanis, C. A. (1982). Antenna Theory Analysis and Design. Harper \& Row Publishers.

[9] Balanis, C. A. (1989). Advanced Engineering Electromagnetics. John Wiley \& Sons.

[10] Wu, T., King, R. (1965). The cylindrical antenna with nonreflecting resistive loading. IEEE Transactions on Antennas and Propagation, 13 (3), 369-373.

[11] Hartansky, R., Bittera, M. (2005). Electromagnetic compatibility em field measurement. In Mechatronika 2005: 8th International Symposium on Mechatronics, May 19-21, 2005. Trenčín, Slovakia: GC TECH, 8487.

[12] Hakim, B., Beard, B., Davis, C. (2009). Precise dielectric property measurements and e-field probe calibration for specificabsorptionrate measurements using a rectangular waveguide. Measurement Science and Technology, 20 (4), 1-9.

[13] Zhou, J., YL, Y. W., Kikuchi, H. (2016). Performance of a MIMO Y-shaped antenna array with electromagnetic vector sensors. Wireless Personal Communications, 86 (3), 1141-1158.

Received June 30, 2016. Accepted November 22, 2016. 